\title{
Research on the influence of the VREs' penetration on the capacity of transmission lines
}

\author{
Ning Chen ${ }^{1,}$, Caixia Wang ${ }^{1}$, Wei Yuan ${ }^{1}$, Qionghui $\mathrm{Li}^{1}$, Xiaoning $\mathrm{Ye}^{1}$, Zhiyong Shi ${ }^{1}$, Qinmiao $\mathrm{Li}^{1} \mathrm{Liang}_{\mathrm{Xu}}{ }^{2}$ and $\mathrm{Hao} \mathrm{Fan}^{3}$ \\ ${ }^{1}$ State Grid Energy Research Institute Co. LTD, Beijing 102209, China \\ ${ }^{2}$ Beijing Power Exchange Center, Beijing 100031, China \\ ${ }^{3}$ State Grid Corporation of China, Beijing 100031, China
}

\begin{abstract}
Under the trend of global carbon emission reduction and energy transformation, variable renewable energies (VREs mainly wind power and solar) will develop rapidly. Many countries have put forward ambitious VREs development plans, and a high penetration of VREs will have a significant impact on the grid. This paper focuses on the analysis of the influence of the VREs' penetration on the capacity of transmission lines, establishes an analysis model based on mixed integer optimization and power transfer distribution factors (FTDFs), and uses this model to carry out a quantitative study on a typical system. Through the analysis of the research results, as the penetration rate of VREs increases, the transmission power of the transmission lines and the investment cost of the transmission lines will increase, and the line utilization rate will decrease. Utility companies should pay attention to the impact of future development of VREs on grid investment costs and recovery.
\end{abstract}

\section{Introduction}

In order to realize the low-carbon transformation of energy, many countries are developing VREs[1-5]. By the end of 2020, the installed wind capacity in China was 281 million kilowatts and solar capacity was 253 million kilowatts. The proportion of VREs' capacity has reached $24.3 \%$. In 2020, 71.67 million kilowatts of wind were added nationwide, 48.2 million kilowatts of solar were installed nationwide, and the annual VREs installed capacity exceed 100 million kilowatts for the first time. In 2020 , the power generation of VREs is 727.5 billion $\mathrm{kWh}$, accounting for $9.7 \%$ of the total electricity consumption in society (penetration rate). China is gradually entering the stage of VREs' high-penetration. As the strategies of "carbon peak and carbon neutrality" and "build a new power system with VREs as the main body" are proposed, VREs will maintain an accelerated development trend.

The output of VREs represented by wind and solar is constrained by resource conditions and has strong randomness and volatility. Expanding the scope of VREs consumption is one of the effective means to stabilize the randomness and volatility of VREs[6]. The cost is to invest more in the transmission network, and some research work has been carried out worldwide.

According to the research of BNEF in the literature [7], in the context of global carbon neutrality, in order to meet the grid connection of renewable energy dominated by VREs, the world needs to increase the investment of power grid by about $\$ 95.2$ trillion from 2020 to 2050 . Literature [8] makes a statistical analysis on the grid cost caused by the wind and solar. The results show that the grid cost related to VREs will increase with the increase of VREs' penetration, and the grid cost of different countries has a certain degree of dispersion. Based on the statistical results in literature [9-10], it is considered that when the penetration rate exceeds a certain value, the cost of power grid will increase linearly with the penetration of VREs. Most of the existing literatures analyze the power grid cost after the VREs is connected to the grid in the form of statistics or empirical value, but there is no literature to carry out systematic quantitative analysis. It is generally believed that the grid cost related to VREs mainly includes the cost of grid connection, transmission network and distribution network. This paper focuses on the impact of VREs on the transmission network.

In this paper, the model of the influence of VREs on transmission line capacity is established based on the power transfer distribution factors (FTDFs) method and mixed integer optimization. Then, the influence of VREs' penetration on transmission line capacity and cost is analyzed based on the model.

\section{Model of VREs' impact on transmission line capacity}

The model takes the minimum system investment and operation cost as the optimization objectives, and takes the system power balance, thermal power output, wind and solar output et al as the constraints. The objective function is:

\footnotetext{
* Corresponding author: ncepuchenning@163.com
} 


$$
\begin{aligned}
& \min Y=\sum_{n=1}^{N} \sum_{s=1}^{S} c_{n, s} H_{n, s}+\sum_{l=1}^{L} c_{l} F_{l}+ \\
& \sum_{t=1}^{T} \sum_{n=1}^{N}\left[\sum_{t h=1}^{T H}\left(o_{n, t h, t} P_{n, t h, t}^{G}+S U C_{n, t h, t}+S D C_{n, t h, t}\right)+\right. \\
& \left.\sum_{s=1}^{S} o_{n, s, t} P_{n, s, t}^{H}+\sum_{p w=1}^{P W} o_{n, p w, t} P_{n, p w, t}\right]
\end{aligned}
$$

Where, $Y$ is the investment and operation cost of the system, $t=1,2,3, \ldots, T$ is the simulation time, $n=1,2$, $3, \ldots, N$ is the bus number, $t h=1,2,3, \ldots, T H$ is the number of thermal power units, $p w=1,2,3, \ldots, P W$ is the number of VREs units, $s=1,2,3, \ldots, S$ is the number of storage units, $l=1,2,3, \ldots, L$ is the number of transmission lines, $c$ represents the initial investment of the corresponding object, $o$ represents the running cost of the corresponding object, $P$ is the power of the corresponding object, $S U C$ is the start-up cost, $S D C$ is the shut-down cost, $H$ is the capacity of storage, $F$ is the transmission power of the line.

The main constraints are as follows.

(1) Node power balance constraints

$$
\begin{aligned}
& L_{n, t}=P_{n, t h, t}+P_{n, s, t}+P_{n, p w, t}+P_{n, h o, t}+ \\
& P_{n, n c, t}+P_{n m, t}
\end{aligned}
$$

Where, $L_{n, t}$ is the load of bus $n$ at time $t$. On the right side of the equal sign is the power of thermal power units, storage units, VREs units, hydropower units, nuclear power units and lines at time $t$.

(2) VREs output constraints

$$
0 \leqslant P_{n, p w, t} \leqslant P_{n, p w, t}^{\max }
$$

Where, $P_{n, p w, t}^{\max }$ is the maximum output of the VREs unit at time $t$.

(3) Startup and shutdown constraints of thermal units

$$
S_{n, t h, \mathrm{t}} \cdot P_{n, t h, t}^{\min } \leqslant P_{n, t h, t} \leqslant S_{n, t h, \mathrm{t}} \cdot P_{n, t h, t}^{\max }
$$

Where, $S_{n, t h, t}$ is the operation state variable of thermal unit, $P_{n, t h, t}^{\min }, P_{n, t h, t}^{\max }$ is the minimum and maximum output of thermal unit.

(4) Startup and shutdown constrains of thermal units

$$
\begin{aligned}
& S U C_{n, s, t} \geqslant s u c_{n, s} \cdot\left(S_{n, t h, t}-S_{n, t h, t-1}\right) \\
& S D C_{n, s, t} \geqslant s d c_{n, s} \cdot\left(S_{n, t h, t-1}-S_{n, t h, t}\right)
\end{aligned}
$$

$s u c_{n, s}, s d c_{n, s}$ are the cost of single start-up and shutdown of thermal unit.

(5) Ramping constraints of thermal units

$-P_{n, t h, t}^{\text {down }} \cdot S_{n, t h, t}-P_{n, t h, t}^{\text {down sd }}\left(S_{n, t h, t-1}-S_{n, t h, t}\right)$

$\leqslant P_{n, t h, t}-P_{n, t h, t-1} \leqslant$

$$
P_{n, t h, t}^{u p} \cdot S_{n, t h, t}+P_{n, t h, t}^{u p s u}\left(S_{n, t h, t}-S_{n, t h, t-1}\right)
$$

Where, $P_{n, t h, t}^{\text {down }}, P_{n, t h, t}^{u p}$ is the limit value of power decrease and increase in unit time, $P_{n, t h, \bar{t}}^{\text {down } s d}, P_{n, \bar{t}, t}^{u p s u}$ are the limit value of power decrease and increase of thermal unit at startup and shutdown time.

(6) Line transmission power constraints

$$
\boldsymbol{P}_{n m, t}=\boldsymbol{P T D F} \cdot \boldsymbol{P}_{n, t}
$$

Where, $\boldsymbol{P}_{\boldsymbol{n} m, \boldsymbol{t}}$ is the line transmission power matrix, $\boldsymbol{P}_{\boldsymbol{n}, \boldsymbol{t}}$ is the net injection power matrix of the bus, PTDF is the power transfer distribution factors matrix.

The derivation of the formula can be referred to the references [11].

(7) VREs utilization constraints

$$
\sum_{n}^{N} \sum_{t}^{T} P_{n, p w, t} \geqslant u r \cdot \sum_{n}^{N} \sum_{t}^{T} P_{n, p w, t}^{\max }
$$

Where, $u r$ is the set utilization rate of VREs, $P_{n, p w, t}^{\max }$ is the theoretical output of VREs unit at each time.

\section{Case calculation results}

Using the above model, a 3-bus system is analyzed. The structure of the system is shown in Fig. 1. Each bus is connected with different loads, plant units and other components, and the lines connecting each component to the bus are ignored. The parameters of each unit are shown in Table 1. The maximum transmission power and electrochemical energy storage power of each line are the optimization results.

\section{Coal 1}

Wind 1

Solar 1

Load 1

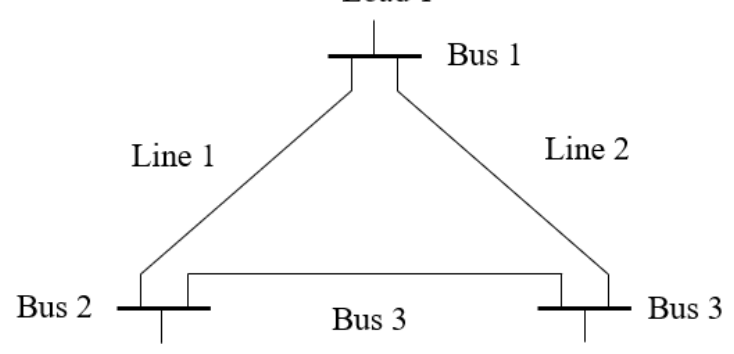

Coal 2

Gas 1

Wind 2

Storage

Solar 2

Load 2

Figure 1. Wiring structure of the studied case.

Table1. Parameters of each element.

\begin{tabular}{|c|c|c|c|}
\hline \multicolumn{4}{|c|}{ Units } \\
\hline Name & $\begin{array}{c}\text { Capacity } \\
\text { (MW) }\end{array}$ & $\begin{array}{c}\text { Minimum } \\
\text { output (p.u.) }\end{array}$ & $\begin{array}{c}\text { Maximum } \\
\text { output (p.u.) }\end{array}$ \\
\hline Coal 1 & 2910 & 0.3 & 1 \\
\hline Coal 2 & 3765 & 0.3 & 1 \\
\hline Gas 1 & 4903 & 0 & 1 \\
\hline \multicolumn{3}{|c|}{ Transmission lines } \\
\hline \multicolumn{3}{|c|}{ Name } & Length (km) \\
\hline Line 1 & 30 \\
\hline \multicolumn{2}{|c|}{ Line 2 } & 20 \\
\hline Line 3 & $11700 \mathrm{MW}$ \\
\hline \multicolumn{2}{|c|}{ Load 1 } \\
\hline Load2
\end{tabular}

The simulation time range is the whole year with 8760 hours. The load, wind power and photovoltaic power curve of a typical day are shown in Figure 2 


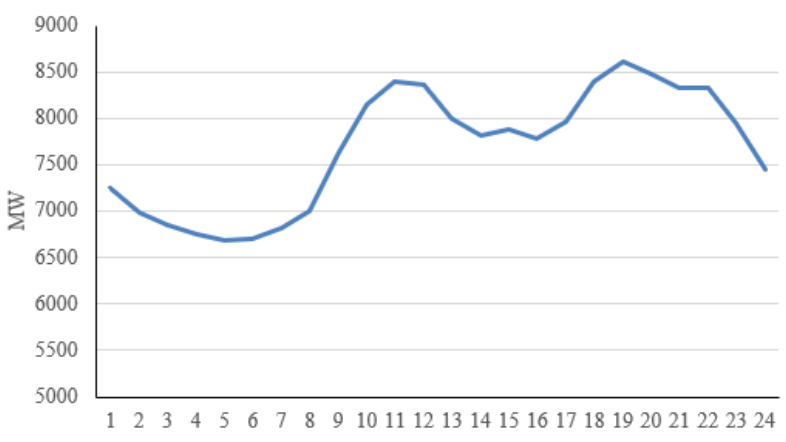

(a) Load curve of typical day

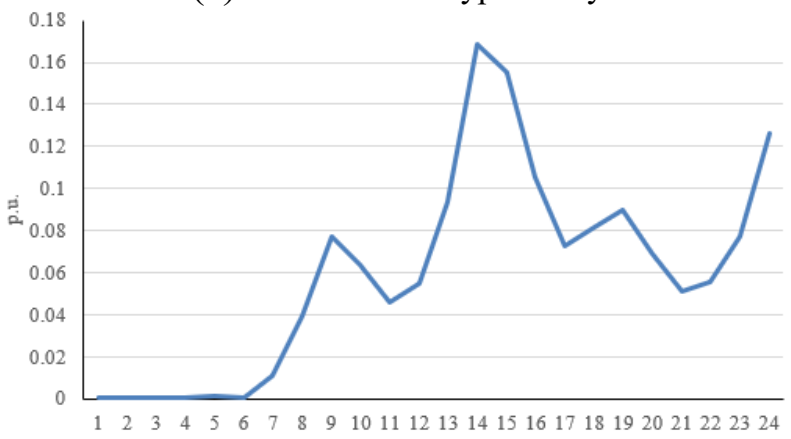

(b) Wind power curve of typical day

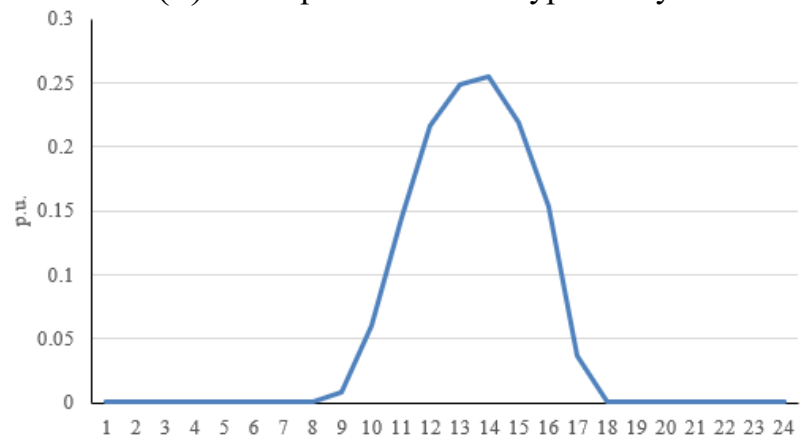

(c) PV power curve of typical day

Figure 2. Used curves of typical day in the simulation

Set the minimum utilization rate of VREs as $90 \%$, gradually increase the capacity of VREs, and get the relationship between the maximum transmission power of each line and the penetration rate of VREs, as shown in Fig. 3. It can be seen from the figure that the maximum transmission power of each line increases with the increase of VREs' penetration. The maximum transmission power of line 1 with penetration of $78 \%$ is nearly 90 times of that with penetration of $29 \%$

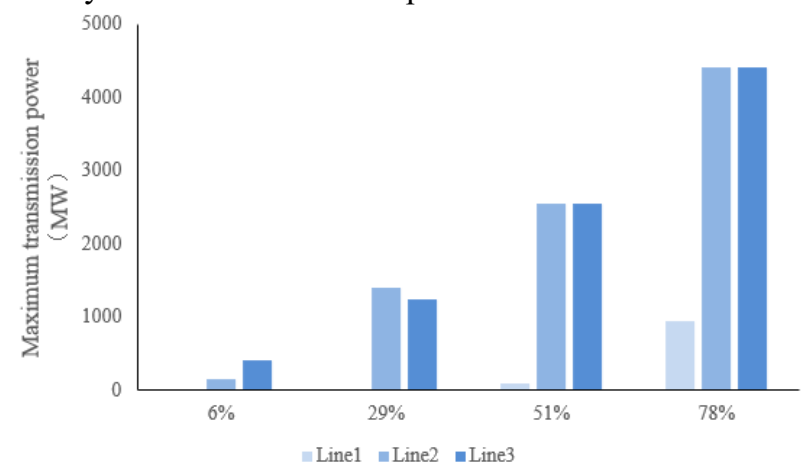

Figure 3. Maximum transmission power of each line under different penetration.

The increase of maximum transmission power also means the increase of line investment. Referring to the investment cost of $500 \mathrm{kV}$ transmission line, the change of line investment cost with penetration of VREs is calculated, as shown in Fig. 4. It can be seen from the figure that the investment cost of the line increases with the increase of penetration, and the investment cost of the line increases $76 \%$ when the penetration is $78 \%$ compared with that of $6 \%$.

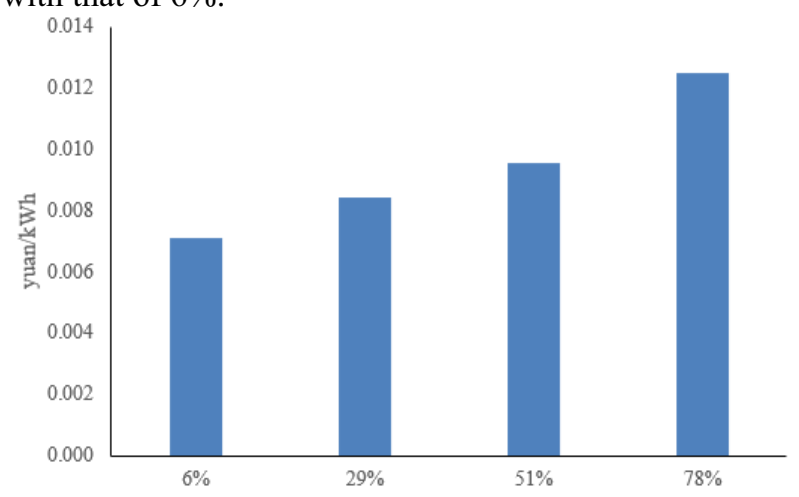

Figure 4. Line investment cost under different penetration.

The equivalent utilization hours of transmission line are defined as the annual transmission power divided by the maximum transmission power. The trend of equivalent utilization hours of the line with the penetration of VREs is shown in Fig. 5. It can be seen from the figure that the equivalent utilization hours of lines decrease with the increase of VREs penetration. When the penetration is $78 \%$, the equivalent utilization hours are only 1943 hours, which is $50 \%$ lower than that when the penetration is $6 \%$. This means that the utilization rate of lines decreases with the increase of VREs penetration, which is not conducive to the cost recovery of utility companies.

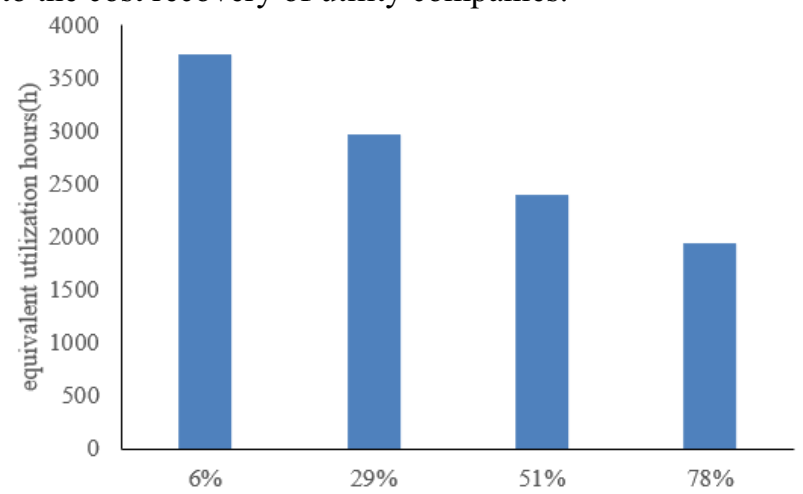

Figure 5. Equivalent utilization hours of the line under different permeability.

The above results are carried out under the premise that the utilization rate of VREs is more than $90 \%$. Fig. 6 shows the maximum transmission power of each line with or without VREs utilization. It can be seen from the figure that under any permeability, the transmission power without utilization constraint is less than that with utilization constraint. Therefore, the utilization rate of VREs is negatively correlated with the transmission power. 


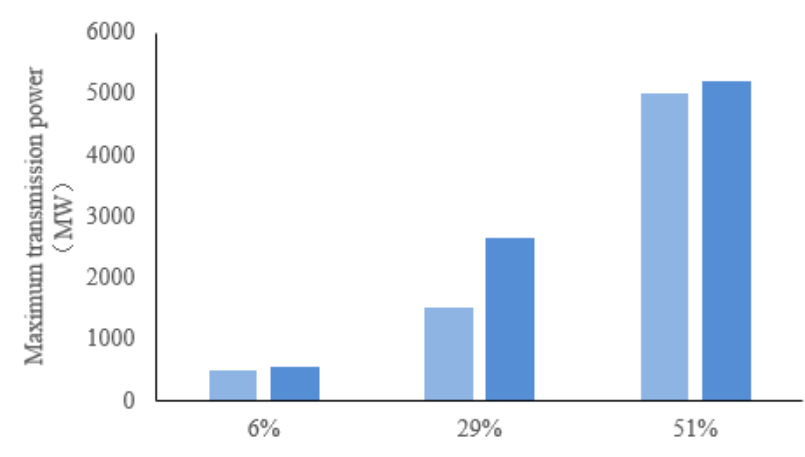

=without VREs utilization constraints = with VREs utilization constraints

Figure 6. Impact of VREs utilization constraints.

\section{Conclusions}

Based on the mixed integer optimization theory and FTDFs method, this paper establishes an analysis model of the impact of VREs on the transmission line capacity, and uses the model to analyze a 3-bus system. The main conclusions are as follows:

1. The power of the transmission lines will increase with the increase of VREs' penetration. In order to realize the high penetration of VREs system, more transmission lines need to be built.

2. The investment cost (yuan/kWh $\mathrm{VRE}$ ) with the increase of VREs' penetration. In the high penetration of VREs system, the related costs of transmission lines will rise significantly.

3. The equivalent utilization hours of transmission lines decrease with the increase of VREs penetration, and the utilization rate of transmission lines decreases with the increase of VREs penetration. Therefore, we must pay attention to the impact of VREs on the cost recovery of utility companies.

4. In the case of utilization constraint, the transmission power of transmission line is greater than that without utilization constraint. In order to ensure the rationality of transmission network investment, the constraints of utilization should be relaxed.

\section{Acknowledge}

This work is supported by State Grid Corporation Science and Technology Project (1400-202057413A-0-0-00).

\section{References}

1. W. Wang, W. Lin, G. He, Enlightenment of 2021 Texas Blackout to the Renewable Energy Development in China, Proceedings of the CSEE 41, (2021)

2. C. Wang, Z. Zheng, Analysis on the Measures and Actions of Germany Power System Operators during Solar Eclipse. Electric Power 48, (2015)

3. $\mathrm{Y} \mathrm{Bu}, \mathrm{X}$ Zhang, On the Way to Integrate Increasing Shares of Variable Renewables in China: Experience from Flexibility Modification and Deep Peak Regulation Ancillary Service Market Based on MILP-UC Programming, Sustainability 13, (2021)
4. Komiyama R, Fujii Y. Large-scale integration of offshore wind into the Japanese power grid, Sustainability Science 1, (2021)

5. J. Hu, B. Huang and D. Du et al. Research on new energy development comprehensive assessment model and application in China, E3S Web Conf, (2019)

6. R. K. Varma, M. Salama, R. Seethapathy and C. Champion. Large-scale photovoltaic solar power integration in transmission and distribution networks, 2009 IEEE Power \& Energy Society General Meeting, Canada (2009)

7. S. Sanghera, Y. Sekine. Power Grid Long-Term Outlook 2021, BloombergNEF (2021)

8. P. J. Heptonstall, R. Gross. A systematic review of the costs and impacts of integrating variable renewables into power grids, Nature Energy 6, (2020).

9. F. Ueckerdt, L. Hirth, et al. System LCOE: What are the costs of variable renewables? ENERGY OXFORD 63, (2013)

10. L. Hirth. The market value of variable renewables. Energy Economics 38, (2013)

11. S. Hagspiel, C. Jaegemann, D. Lindenberger, et al. Cost-optimal power system extension under flowbased market coupling, Energy 66, (2014) 